\title{
Medical retina hotline: Service evaluation and assessment of efficacy of a novel system in a tertiary care hospital
}

\author{
Aditi Ashok Kumar Agarwal $\mathbb{1}^{1} \cdot$ Ricardo Alberto Romero Fontenlos $^{1} \cdot$ Deepthy Menon $^{1} \cdot$ Robin Hamilton $^{1}$
}

Received: 22 January 2021 / Revised: 27 January 2021 / Accepted: 18 February 2021 / Published online: 10 March 2021

(c) Crown 2021

\section{To the Editor:}

The COVID-19 pandemic-related social distancing made it imperative to control patient flow to hospitals to minimise spread of the virus, therefore switching to tele-consultations and telephone enquiry lines were practical strategies applied broadly across the UK [1-3]. Centres for Disease Control and Prevention recommended telemedicine in lieu of live clinic visits in anticipation of an extended period of social distancing [4]. The guidelines for telemedicine were laid down by Royal College of Ophthalmologists in May 2020 (https://www.rcophth.ac.uk/wp-content/uploads/2020/06/ Overview-of-digital-technology-and-telemedicine-forcovid-060520-1.pdf). However, the effectiveness and efficiency of these tele-consultations were unclear.

We undertook a survey of clinicians who were delegated to maintain the newly developed telephone hotline where patients actively sought advice for medical retinal problems within a leading provider of eye health services in the UK. The aim of this study is to objectively assess the efficacy of a telephone helpline consultation service.

This prospective observational study was performed in the medical retina unit as an audit (CA20/MR/638) within a tertiary care hospital in June 2020. All tele-consultations were completed by three medical retina fellows. The first step was to design a questionnaire that could assess the basic tenets of what an effective tele-consultation means (Fig. 1).

Only follow-up patients who contacted the medical retina phone line for a clinical enquiry were eligible for the present study. New patients and patients seeking assistance for administrative issues were excluded. All patients included in the study deliberately contacted our phone line, and

Aditi Ashok Kumar Agarwal

aditiagarwal.26888@gmail.com

Moorfields Eye Hospital NHS Foundation Trust, London, UK therefore provided their consent to participate in teleconsultations.

The telephone consultation followed a format designed to: clarify the patients complains, review the medical retina concerns, consider relevant comorbidity and previous ophthalmic treatment and make suggestions for future management. Following the consultation, the ophthalmology specialist mailed the summary to the patient, along with an appropriate management plan. The specialist then completed the questionnaire, and the data were transferred to an Excel spreadsheet. The term 'working diagnosis' meant that the symptoms described by the patient partially fit the previous diagnosis of the patient and the clinician could make a judgement as regards to the possible underlying pathophysiology.

If the ophthalmologist considered it necessary or if patients' issues persisted, patients were booked for a faceto-face assessment to better ascertain the diagnosis and refine the management recommendations and solve the problem. Finally, outcomes for those patients who attended a face-to-face consultation were registered (Fig. 2).

A total of 110 consecutive telephone queries were answered by three medical retina specialists over the period of 1 month. A total of 33 queries related to administrative and non-clinical issues were excluded and the 77 remaining tele-consultations were included in this analysis. The mean age of the patient was 71 (SD 16.2 years, range 29-92 years) and $54(70.1 \%)$ were females.

The most common working diagnosis of patients who participated in the tele-consultations were choroidal neovascularization due to age-related macular degeneration (31\%) (Fig. 3).

The absolute need for clinical examination was thought to be required in $21 / 77$ (28\%) patients and was not required in $11 / 77$ (14\%) cases. In 27/77 (35\%) patients, clinical examination would have helped in contributing to the treatment plan (Fig. 4).

A definite diagnosis could be reached in 22/77 (29\%) cases, while in 7/77 (9\%) no clear diagnosis could be made. 
In about $48 / 77$ (62\%) cases, the queries matched with the working diagnosis. The clinicians unanimously agreed (88\% of responses) that an investigation done remotely would have aided their diagnosis and management plan. The most frequent investigation (59\%) needed was thought to be an optical coherence tomography (Fig. 5).

Two-third of the patients 51/77 $(66.2 \%)$ were called in for a face-to-face appointment for better diagnosis or to make an appropriate treatment plan or to perform an intervention. Active surveillance was continued for the remaining 26/77 (33.7\%) and they remained stable until their next appointment.

Of the 51/77 patients who had face-to-face consultations, 22/51 (43\%) needed laser or injection therapy, 20/51 (39\%)

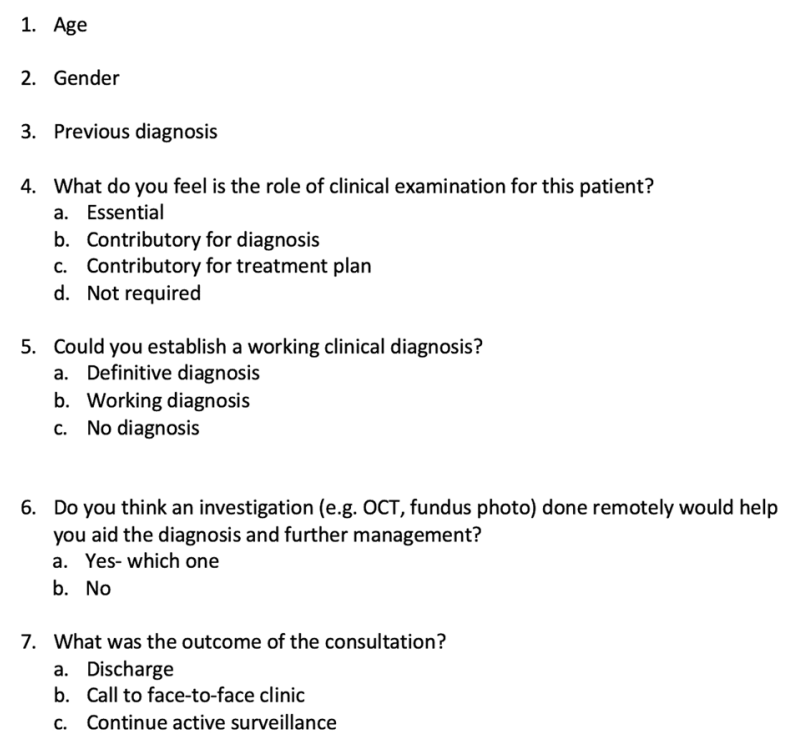

Fig. 1 Hotline service evaluation questionnaire. Completed after tele-consultation by Specialist. needed no treatment and $9 / 51(18 \%)$ chose not to attend the clinic (Fig. 2). Out of the $31 / 51$ patients who were called to hospital with an intention of possibly injecting them, 10/31 (32\%) patients did not need an injection and $7 \%$ patients did not attend the clinic.

Our study reveals some interesting results in a subspeciality that relies so heavily on imaging and biomicroscopy. The majority of the patients who contacted the medical retina services during the lockdown period were the ones who were on ongoing intravitreal anti-VEGF injection therapy. Definitive diagnosis and treatment plan could be achieved in about $1 / 3$ rd patients through remote consultations but $2 / 3 \mathrm{rd}$ of the patients $(66.2 \%)$ required a face-toface visit as the clinicians were unable to make an accurate diagnosis and the patients required OCT or other investigations to decide a management plan or required an intervention. When we reviewed the outcome of the patients who attended face-to-face consultation, $39 \%$ did not require any intervention. It could be deemed that these patients made an 'unnecessary trip' to the hospital. Thirty-two percent (10/31) patients who were advised that they required intravitreal therapy based on their telephone consultation did not need an injection following face-to-face consultation. This again highlights the need for community-based diagnostic clinics where patients can go locally and have their visual acuity tested, OCT and retinal colour photographs done and reported virtually to them by telephone consultations. In addition, the 26/77 (33.7\%) patients who were kept on active surveillance remained stable until the next appointment and were saved an unnecessary trip to the hospital. This number could be even higher if the diagnostic facilities were made widely available. These can be located at GP practices, local optometrists or stand-alone facilities can be built to reduce the pressures in retinal clinics.
Fig. 2 Flowchart of the teleconsultation evaluation. Number of tele-consultatios and their outcomes.

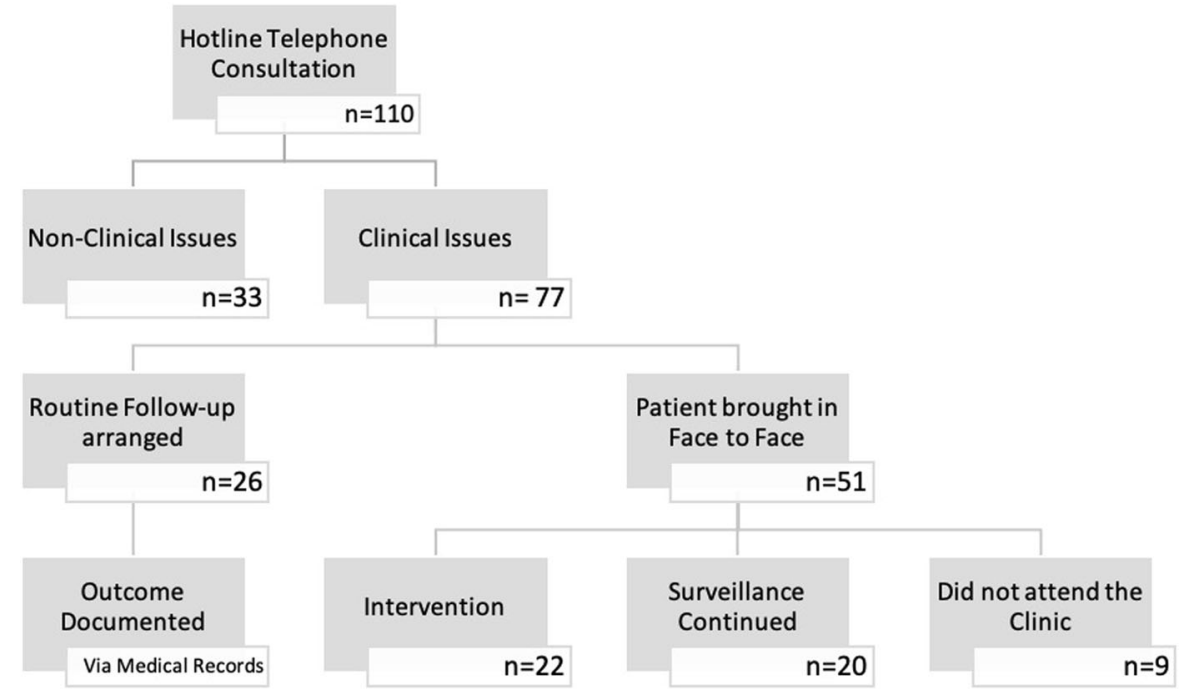




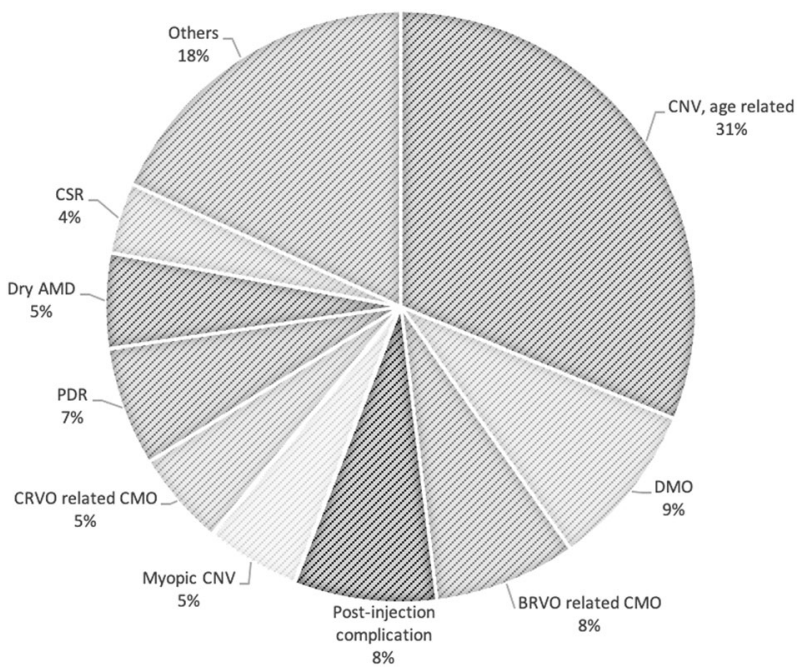

Fig. 3 Distribution of pationts. Pie-diagram showing distribution of follow-up patients according to their previous diagnosis.

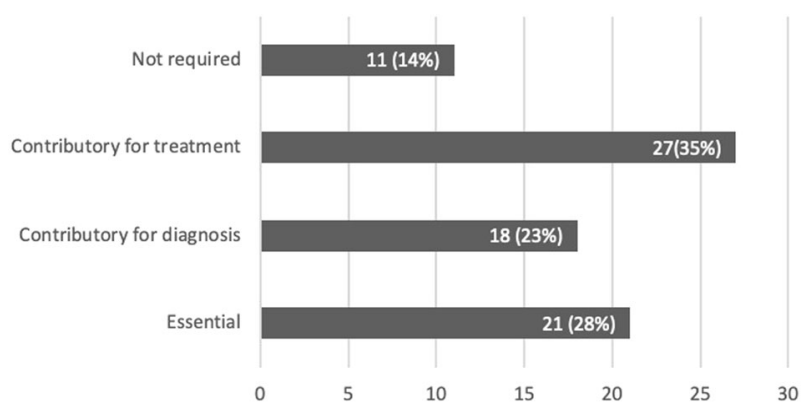

Fig. 4 Role of clinical examination. Line graph showing the role of clinical examination felt at the time of tele-consultation.

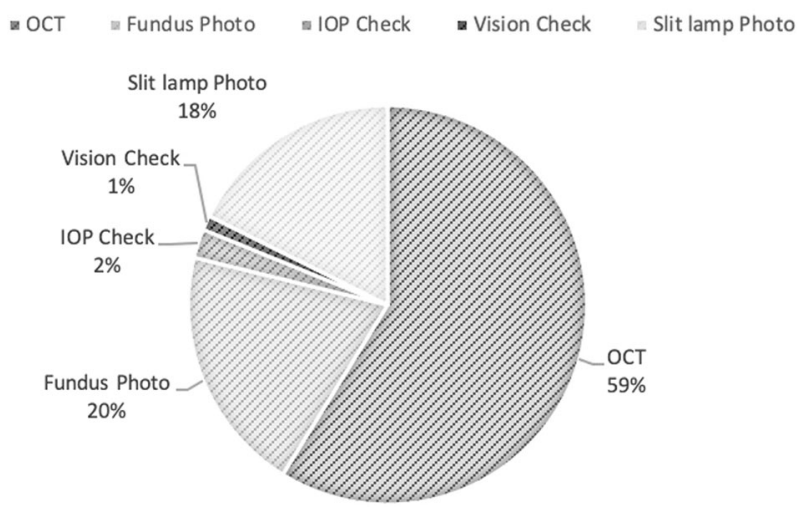

Fig. 5 Remote investigations. Pie-diagram showing distribution of need for various imaging modalities if done remotely would add value to the tele-consultation evaluation.

Kelly et al. managed 50 patients with macular oedema remotely in partnership with the community optometrist [5]. The optometrist assessed the patients, performed an OCT and transferred the images to the hospital using a secure and compliant pathway, which were then assessed by the ophthalmologist and the patients were informed if an intervention was deemed necessary.

Various home-based visual function monitoring technology platforms are now available, which offer clinically validated functional tests like Snellen's chart, Amsler's Grid, contrast sensitivity and low luminescence acuity $[6,7]$. Home-based technician-free OCT devices are also currently under development.

Our study does have some limitations. There could be an element of subjectivity and inter-observer bias amongst different clinicians answering the helpline. Although most patients were pleased with availability of this clinician-led service, the patient satisfaction and feedback were not objectively evaluated.

We believe that provision of a hotline service along with development and validation of a pathway for diagnostic or monitoring facilities have multiple benefits such as reduced risk of exposure to hospital visits, increased compliance with the social distancing norms and ensuring patients needing urgent care receive treatment in a timely fashion despite restrictions induced by the pandemic.

The provision of a clinician-led hotline service is indeed useful for a group of patients who require urgent advice against the backdrop of the COVID-19 pandemic. The development of a pathway for active remote monitoring would further increase the efficacy of the service.

Author contributions The manuscript has been read and approved by all the authors and the requirements for authorship have been met and the manuscript represents honest work.

\section{Compliance with ethical standards}

Conflict of interest The authors declare no competing interests.

Ethics approval Ethics approval from the institutional ethics board was waived because it was done as an audit.

Publisher's note Springer Nature remains neutral with regard to jurisdictional claims in published maps and institutional affiliations.

\section{References}

1. Adlhoch C, Baka A, Ciotti M, Gomes J, Kinsman J, Leit-meyer K, et al. Considerations relating to social distancing measures in response to the COVID-19 epidemic. European Centre for Disease Prevention and Control. 2020. https://www.ecdc.europa.eu/en/ publications-data/considerations-relating-social-distancing-measuresresponse-covid-19-second.

2. National Center for Immunization and Respiratory Diseases (NCIRD), Division of Viral Diseases. CDC's implementation of mitigation strategies for communities with local COVID-19 transmission. 2020. https://www.cdc.gov/coronavirus/2019-ncov/dow nloads/community-mitigation-strategy.pdf.

3. Xia W, Liao J, Li C, Li Y, Qian X, Sun X, et al. Transmission of coronavirus disease 2019 during the incubation period may lead to 
a quarantine loophole. medRxiv 2020. https://doi.org/10.1101/ 2020.03.06.20031955.

4. Centers for Disease Control and Prevention. Get your clinic ready for coronavirus disease 2019 (COVID-19). https://www.cdc.gov/corona virus/2019-ncov/hcp/clinicpreparedness.html. Accessed 18 Apr 2020.

5. Kelly SP, Wallwork I, Haider D, Qureshi K. Teleophthalmology with optical coherence tomography imaging in community optometry. Evaluation of a quality improvement for macular patients. Clin Ophthalmol. 2011;5:1673-8.
6. AREDS2-HOME Study Research Group, Chew EY, Clemons TE, Bressler SB, Elman MJ, Danis RP, et al. Randomized trial of a home monitoring system for early detection of choroidal neovascularization home monitoring of the Eye (HOME) study. Ophthalmology. Ophthalmology. 2014;121:535-44.

7. Holekamp NM. Moving from clinic to home: what the future holds for ophthalmic telemedicine. Am J Ophthalmol. 2018;187: xxviii-xxxv. https://doi.org/10.1016/j.ajo.2017.11.003. 